\title{
Contribuição ao estudo das características físico-químicas e da fração lipídica do leite orgânico
}

\author{
Contribution to the study of physicochemical characteristics and lipid fraction of organic milk \\ Michele Gabriela Nogueira FANTI ${ }^{1}$, Keila Emílio de ALMEIDA ${ }^{1}$, Alexandre Mariani RODRIGUES ${ }^{1}$, \\ Roberta Claro da SILVA ${ }^{1}$, Ana Carolina Rodrigues FLORENCE ${ }^{1}$, Luiz Antônio GIOIELLI ${ }^{1}$, \\ Maricê Nogueira de OLIVEIRA ${ }^{1 *}$
}

\begin{abstract}
Resumo
Produtos orgânicos consistem em uma alternativa para uma alimentação saudável. Considera-se leite orgânico aquele produzido em sistema no qual é vedado o uso de agrotóxico sintético ou outros insumos artificiais tóxicos e organismos geneticamente modificados, visando à oferta de produtos saudáveis e de elevado valor nutricional. Existem poucos dados disponíveis sobre leite orgânico na literatura, mas há indicações de que este apresenta maior teor nutritivo quando comparado ao leite produzido em sistema convencional. O objetivo deste trabalho foi caracterizar a composição físico-química, o perfil de ácidos graxos e ácido linoléico conjugado (CLA) em leites pasteurizados integrais oriundos de agricultura orgânica brasileira. Os leites orgânicos foram analisados durante o período de doze meses e os resultados comparados com aqueles obtidos de leites pasteurizados integrais provenientes de sistemas convencionais. A sazonalidade e o manejo dos animais afetaram a composição química dos leites estudados no período de doze meses. Os teores de proteína foram maiores em leite orgânico, porém tendência inversa foi observada nos níveis de gordura. Os principais ácidos graxos do leite não apresentaram diferenças significativas durante as estações do ano e entres os leites. Entretanto, foi verificado maior teor de CLA em leites orgânicos (2,8 vezes maior que em leite convencional), provavelmente devido à dieta dos animais.
\end{abstract}

Palavras-chave: leite; agricultura orgânica; composição; ácido linoléico conjugado.

\begin{abstract}
Organic milk is produced in an agro-system in which the use of synthetic livestock additives or other artificial inputs are forbidden. The objective of this work was to characterize the physicochemical composition and the profile of fatty acids and conjugated linoleic acid (CLA) in pasteurized whole milk, a product deriving from organic agriculture in Brazil. The organic milk was analyzed during the period of twelve months, and the results were compared with those of milk obtained from conventional production systems. The seasonality and the handling of the animals affected the chemical composition of the studied milk during the one-year period. The protein contents were higher in the organic milk, but the contrary was observed for the fat levels. The main fatty acids of the milk did not present significant differences during the seasons of the year, and in the milk studied. However, higher amounts of CLA were found in organic milk (2.8 times higher than in conventional milk). This is probably due to animal diets.

Keywords: milk; organic; composition; conjugated linoleic acid.
\end{abstract}

\section{Introdução}

Nas últimas décadas, o interesse por alimentos puros vem crescendo, impulsionando o mercado a apontar soluções para os atuais problemas de saúde causados pelo alto consumo de alimentos contaminados por metais pesados, agrotóxicos, antibióticos e hormônios. Os alimentos provenientes de agricultura orgânica vêm se destacando como alternativa para uma alimentação saudável.

Agricultura orgânica é um termo definido por padrões da IFOAM (International Federation of Organic Agriculture Movements) e toda produção e todo processamento de alimento orgânico obedecem a um rigoroso conjunto de normas e diretrizes. Desta maneira, considera-se leite orgânico aquele produzido em sistema no qual é vedado o uso de agrotóxico sintético, antibióticos ou outros insumos artificiais tóxicos e organismos geneticamente modificados, visando à oferta de produtos saudáveis e de elevado valor nutricional (BRASIL, 2003).

De acordo com os princípios da agricultura orgânica, a atividade animal deve estar, tanto quanto possível, integrada à produção vegetal, visando à otimização da reciclagem dos nutrientes (dejetos animais e biomassa vegetal), menor dependência de insumos externos (rações e volumosos) e potencialização de todos os benefícios diretos e indiretos advindos dessa integração (DAROLT, 2001). A alimentação dos bovinos e de outros animais deve ser realizada de acordo com as normas de agricultura orgânica e conter no mínimo 90\% de alimentos orgânicos provenientes da fazenda. A produção de volumosos e

Recebido para publicação em 27/9/2007

Aceito para publicação em 4/3/2008 (002884)

Departamento de Tecnologia Bioquímico Farmacêutica, Faculdade de Ciências Farmacêuticas, Universidade de São Paulo - USP, Av. Prof. Lineu Prestes, 580,

CEP 05508-000, São Paulo - SP, Brasil, E-mail: monolive@usp.br

${ }^{*}$ A quem a correspondência deve ser enviada 
concentrados deve ser feita por meio da formação e manejo das pastagens, capineiras, silagem e feno. A oferta de material verde fresco como hortaliças, rami, guandu e gramíneas é indicada para complementar a alimentação dos animais, pois, dentre outros fatores, exerce influência direta na composição do leite. Deste modo, o leite orgânico pode apresentar características diferentes daquele obtido de animais criados em pecuária leiteira convencional (BOREL; SØRENSEN, 2004).

Segundo Rosati e Aumaitre (2004), poucos dados experimentais comparando características biológicas, tecnológicas e econômicas sobre sistemas convencional e orgânico estão disponíveis na literatura e são, muitas vezes, contraditórios (LUUKONEN et al., 2005). Alimentos de origem orgânica apresentam, em média, mais sais minerais, antioxidantes, $\alpha$-tocoferol, $\beta$-caroteno, vitamina $C$ e fitonutrientes, entre outros (HEATON, 2001; FELSOT; ROSEN, 2004; CARIS-VEYRAT et al., 2004). Toledo et al. (2002) analisaram leites crus orgânicos e convencionais no período de um ano, na Suécia, e não encontraram diferenças significativas para composição média entre os produtos, concluindo, porém, que esses resultados poderiam ser diferentes em outros países ou regiões.

Dentre os componentes do leite, a gordura esteve durante anos associada a uma variedade de doenças humanas, devido a seu alto conteúdo de ácidos graxos saturados (BERGAMO et al., 2003). Recentes estudos, porém, têm evidenciado componentes saudáveis da gordura láctea, tais como o ácido linoléico conjugado (CLA). O CLA consiste em um grupo de ácidos graxos encontrado predominantemente no leite e na carne de animais ruminantes, como bovinos, ovinos e caprinos (PRANDINI et al., 2007). Cerca de $90 \%$ do CLA em gorduras de ruminantes é representado pelo 9-cis,11-trans-CLA (KÜHLSEN et al., 2005). O teor de CLA na gordura do leite geralmente se encontra entre $0,3 \mathrm{e}$ $1,0 \%$. No entanto, vários fatores podem influenciar o conteúdo de CLA, como as estações do ano, a alimentação e a raça do animal, o estágio de lactação e o processamento do alimento (JAHREIS et al., 1997; SEBÉDIO et al., 1999; PRANDINI et al., 2007). O CLA tem sido amplamente pesquisado por suas propriedades anticarcinogênicas e capacidade de reduzir a gordura corporal enquanto aumenta, concomitantemente, a massa muscular. Outros efeitos benéficos atribuídos ao consumo do CLA são a proteção contra aterosclerose, caquexia e o desenvolvimento de diabetes (CAMPBELL et al., 2003; MATTILA-SANDHOLM; SAARELA, 2003). Bergamo et al. (2003) observaram teores significativamente maiores de ácido linolênico, $\alpha$-tocoferol, $\beta$-caroteno e CLA em leite e produtos lácteos orgânicos, quando os compararam com produtos convencionais.

Jahreis et al. (1997) compararam a composição de ácidos graxos em leite integral orgânico com aqueles provenientes do sistema convencional a pasto e confinado. O leite orgânico apresentou maior conteúdo de CLA - 0,80\% do total de ácidos graxos em comparação com $0,34 \%$ do convencional confinado e $0,61 \%$ do sistema convencional a pasto. Estes resultados confirmam os de Bergamo et al. (2003). De acordo com estes autores, a razão para o maior conteúdo de CLA no leite orgânico se deve ao maior teor de ácidos graxos poliinsaturados presentes na dieta de animais manejados nesse sistema. Este permitiria a possibilidade de maior formação de CLA pela biohidrogenação das bactérias ruminais. Outra razão seria o maior teor de fibras da dieta orgânica que também influencia a biohidrogenação, produzindo maiores concentrações de CLA (JAHREIS et al., 1997; BERGAMO et al., 2003).

No Brasil, existem atualmente poucos produtores de leite orgânico e a literatura dispõe de dados escassos sobre a característica do leite orgânico produzido no País. Sendo assim, o objetivo deste trabalho é o de contribuir para o estudo das características do leite orgânico, analisando a composição físico-química e o perfil de ácidos graxos e de ácido linoléico conjugado em leites pasteurizados integrais oriundos de agricultura orgânica, comercializados na cidade de São Paulo ao longo de doze meses.

\section{Material e métodos}

\subsection{Material}

Foram utilizados três leites pasteurizados integrais provenientes de agricultura orgânica $(\mathrm{OA}, \mathrm{OB}$ e $\mathrm{OC})$ e três leites pasteurizados integrais de origem convencional (CD, CE e CF) obtidos no comércio local na cidade de São Paulo.

\subsection{Métodos}

\section{Análises físico-químicas}

Foram realizadas análises físico-químicas - densidade, acidez total, extrato seco, gordura, proteína, lactose e cinzas, e determinados o perfil de ácidos graxos e o teor de ácido linoléico conjugado. As análises foram realizadas em triplicata no período de junho de 2006 a maio de 2007.

Densidade, acidez total, gordura e lactose foram determinadas segundo os métodos descritos em BRASIL. Ministério da Agricultura, Pecuária e Abastecimento (2006). A determinação do extrato seco foi realizada em estufa com circulação forçada de ar a $100-105{ }^{\circ} \mathrm{C}$, até peso constante, seguindo-se o método recomendado pela AOAC (1995). A proteína bruta foi calculada em função dos teores de nitrogênio total determinado pelo método de micro Kjeldahl, multiplicado pelo fator 6,38 (AOAC, 1995). A fração cinza foi obtida gravimetricamente pela determinação da perda de peso do material a $550{ }^{\circ} \mathrm{C}$, segundo o método preconizado pelo Instituto Adolfo Lutz (1985). Os resultados foram expressos em porcentagem.

Análise de ácidos graxos e determinação de ácido linoléico conjugado

\section{Preparação da amostra}

A extração da gordura do leite para posterior quantificação de ácidos graxos foi realizada segundo as normas da International Organization For Standardization. ISO/DIS (2000), método 14156.

Preparação de ésteres metílicos

A preparação de ésteres metílicos para posterior quantificação de ácidos graxos foi realizada segundo as normas da 
International Organization For Standardization. ISO/DIS (2000), método 15884.

Análise cromatográfica

A análise de ácidos graxos foi realizada por cromatografia em fase gasosa, segundo as normas da AOCS (1997), método Ce 1-62, em cromatógrafo a gás Varian GC, modelo 3400 CX (Varian, EUA), equipado com detector de ionização de chama. Foi utilizada coluna capilar de sílica fundida CP WAX 52 CB (Chrompack, EUA), com 30 metros de comprimento $\times 0,25 \mathrm{~mm}$ de diâmetro interno e contendo $0,25 \mu \mathrm{m}$ de polietilenoglicol dentro da coluna. As condições foram:

- Injeção split, razão de 50:1;

- Temperatura da coluna: $75^{\circ} \mathrm{C}$ por 3 minutos, programada até $150^{\circ} \mathrm{C}$ numa razão de $37,5^{\circ} \mathrm{C} /$ minutos e novamente programada até $215^{\circ} \mathrm{C}$ a $3^{\circ} \mathrm{C} /$ minutos;

- Gás de arraste: hélio, numa vazão de 1,5 mL/minutos;

- Gás make up: hélio a $30 \mathrm{~mL} / \mathrm{minutos}$

- Temperatura do injetor $250{ }^{\circ} \mathrm{C}$; e

- Temperatura do detector $280^{\circ} \mathrm{C}$.

A composição qualitativa foi determinada por comparação dos tempos de retenção dos picos com os respectivos padrões de ácidos graxos. A composição quantitativa foi realizada por normalização de área, sendo expressa como porcentagem em massa (DÍAZ GAMBOA; GIOIELLI, 2003).

\subsection{Análise estatística}

Os resultados foram submetidos à Análise de Variância (ANOVA) e teste de Tukey para comparação de médias utilizando-se o programa Statistica 6.0 (Statsoft, Tulsa, USA), considerando $\mathrm{p}<0,05$.

\section{Resultados e discussão}

\subsection{Variação sazonal da composição físico-química}

Os resultados da composição físico-química de leites integrais orgânicos e convencionais comercializados na cidade de
São Paulo avaliados ao longo de 12 meses no período de junho de 2006 a maio de 2007 estão apresentados na Tabela 1.

A densidade do leite, relação entre seu peso e volume, é normalmente medida a $15^{\circ} \mathrm{C}$ ou corrigida para essa temperatura, apresentando valores entre 1,023 e 1,040 g. $\mathrm{mL}^{-1}$ em leites normais. Seu valor é diretamente influenciado pelo conteúdo de gordura e de sólidos não-gordurosos. Sendo assim, um leite com teor de gordura baixo ou nulo, como o leite desnatado, apresenta densidade maior, sendo que, no leite integral, a gordura contribui para valores mais baixos (AMIOT, 1991). Observou-se que os leites analisados neste estudo não apresentaram irregularidades, mas variações durante as estações do ano que, apesar de significativas $(\mathrm{p}<0,05)$, foram pequenas (Tabela 1). Em leite orgânico, a densidade variou em média de $1,030 \mathrm{~g} / \mathrm{cm}^{3}$ (verão de 2007) a 1,032 g/ $\mathrm{cm}^{3}$ (primavera de 2006). Durante o outono e inverno, os valores foram intermediários $\left(1,031 \mathrm{~g} / \mathrm{cm}^{3}\right)$. Tendência oposta foi observada em relação a este parâmetro em leites convencionais, cuja variação foi de $0,001 \mathrm{~g} / \mathrm{cm}^{3}$.

A acidez Dornic variou significativamente em leite orgânico durante as estações do ano (Tabela 1). Observou-se que, durante o verão, a acidez média foi mais alta que nos outros períodos $\left(16,78^{\circ} \mathrm{D}\right)$. Este fato pode ser explicado pelo aumento da temperatura ambiente, que contribui diretamente no parâmetro analisado. Todos os componentes capazes de combinar com os íons básicos contribuem para a acidez do leite. Este equilíbrio entre os constituintes básicos (sódio, potássio, magnésio, cálcio e hidrogênio) e os ácidos (fosfatos, carbonatos, hidróxidos e proteínas) determina a acidez do leite. Em temperaturas elevadas, o fosfato tricálcio pode precipitar e produzir aumento da acidez devido à dissociação dos radicais fosfatos. $\mathrm{O}$ calor também pode decompor a lactose em diversos ácidos orgânicos e neutralizar os aminogrupos das proteínas (AMIOT, 1991). O leite convencional apresentou valores menores de acidez (Tabela 1) e pouca variabilidade durante as estações do ano, sendo em média $15,61^{\circ} \mathrm{D}$.

Observa-se, conforme a Tabela 1, que, durante a primavera, o leite orgânico apresentou $12,12 \%$ de extrato seco, sendo significativamente maior $(\mathrm{p} \leq 0,05)$ que aquele encontrado no verão $(11,28 \%)$. Em leite convencional, não foi encontrada diferença estatística entre as amostras. Em períodos de calor, ocorre

Tabela 1. Composição ${ }^{1}$ físico-química de leites integrais orgânicos e convencionais comercializados na cidade de São Paulo.

\begin{tabular}{|c|c|c|c|c|c|c|c|c|}
\hline & & $\begin{array}{c}\text { Densidade } \\
\left(\mathrm{g} / \mathrm{cm}^{3}\right)\end{array}$ & $\begin{array}{c}\text { Acidez } \\
\left({ }^{\circ} \text { Dornic }\right)\end{array}$ & $\begin{array}{c}\text { Extrato seco } \\
(\%)\end{array}$ & $\begin{array}{c}\text { Gordura } \\
(\%)\end{array}$ & $\begin{array}{c}\text { Proteína } \\
(\%)\end{array}$ & $\begin{array}{c}\text { Lactose } \\
(\%)\end{array}$ & $\begin{array}{c}\text { Cinzas } \\
(\%)\end{array}$ \\
\hline \multirow[t]{4}{*}{ Leite orgânico } & Inverno/06 & $1,031^{\mathrm{abc}} \pm 0,001$ & n.d. & $11,53^{\mathrm{ab}} \pm 0,95$ & $2,67^{\mathrm{a}} \pm 0,69$ & $3,31^{\mathrm{cd}} \pm 0,16$ & $4,42^{\mathrm{a}} \pm 0,30$ & $0,80^{b} \pm 0,12$ \\
\hline & Primavera/06 & $1,032^{c} \pm 0,001$ & $15,81^{\mathrm{ab}} \pm 1,08$ & $12,12^{\mathrm{b}} \pm 0,79$ & $3,30^{\mathrm{b}} \pm 0,66$ & $3,24^{\mathrm{bc}} \pm 0,12$ & $4,52^{\mathrm{a}} \pm 0,16$ & $0,72^{\mathrm{a}} \pm 0,03$ \\
\hline & Verão/07 & $1,030^{\mathrm{ab}} \pm 0,001$ & $16,78^{\mathrm{b}} \pm 1,46$ & $11,28^{\mathrm{a}} \pm 0,45$ & $3,03^{\mathrm{ab}} \pm 0,62$ & $3,22^{b c} \pm 0,16$ & $4,39^{a} \pm 0,18$ & $0,74^{\mathrm{a}} \pm 0,03$ \\
\hline & Outono/07 & $1,031^{\mathrm{abc}} \pm 0,001$ & $15,73^{\mathrm{ab}} \pm 1,79$ & $11,73^{\mathrm{ab}} \pm 1,03$ & $3,32^{\mathrm{b}} \pm 0,83$ & $3,42^{\mathrm{d}} \pm 0,20$ & $4,44^{\mathrm{a}} \pm 0,19$ & $0,73^{a} \pm 0,05$ \\
\hline \multirow[t]{4}{*}{ Leite convencional } & Inverno/06 & $1,030^{\mathrm{a}} \pm 0,002$ & n.d. & $11,32^{\mathrm{a}} \pm 1,00$ & $3,39^{b} \pm 0,29$ & $3,01^{\mathrm{a}} \pm 0,23$ & $4,37^{\mathrm{a}} \pm 0,30$ & $0,70^{\mathrm{a}} \pm 0,03$ \\
\hline & Primavera/06 & $1,031^{\mathrm{abc}} \pm 0,000$ & $15,47^{a} \pm 1,01$ & $11,75^{\mathrm{ab}} \pm 0,28$ & $3,39^{b} \pm 0,22$ & $3,07^{\mathrm{ab}} \pm 0,10$ & $4,48^{\mathrm{a}} \pm 0,13$ & $0,71^{a} \pm 0,02$ \\
\hline & Verão/07 & $1,030^{\mathrm{ab}} \pm 0,001$ & $15,61^{\mathrm{ab}} \pm 1,03$ & $11,72^{\mathrm{ab}} \pm 0,29$ & $3,49^{\mathrm{b}} \pm 0,12$ & $3,11^{\mathrm{ab}} \pm 0,20$ & $4,40^{\mathrm{a}} \pm 0,16$ & $0,70^{\mathrm{a}} \pm 0,02$ \\
\hline & Outono/07 & $1,031^{\mathrm{abc}} \pm 0,001$ & $15,75^{\mathrm{ab}} \pm 1,15$ & $11,73^{\mathrm{ab}} \pm 0,50$ & $3,47^{\mathrm{b}} \pm 0,31$ & $3,19^{b c} \pm 0,19$ & $4,37^{\mathrm{a}} \pm 0,24$ & $0,72^{a} \pm 0,03$ \\
\hline
\end{tabular}

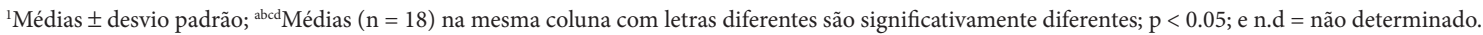


diminuição de ingestão de alimentos por parte dos animais, acompanhada por aumento na ingestão de água. A alimentação restringe-se, basicamente, à pastagem, que apresenta maior teor de água em sua composição, já que há maior incidência de chuvas. Esses fatores provocam diluição dos sólidos do leite. Por outro lado, durante períodos de baixas temperaturas, o maior requerimento de energia para a manutenção da temperatura corporal acarreta redução no aporte energético necessário à produção de leite, levando à conseqüente diminuição na produção. Além disso, a associação de baixas temperaturas com o tipo de alimentação fornecida ao gado nessa situação (menor concentração de fibras) provoca elevação relativa dos sólidos totais (HOLMES; WILSON, 1989).

Verificou-se a influência da sazonalidade sobre o leite orgânico no parâmetro de teor de gordura, como pode ser observado na Tabela 1. Segundo Harding (1995) e Block (2000), o teor de gordura no leite varia em razão de diferenças entre raças e estágio da lactação, tornando esta variável mais instável que os percentuais de proteína e lactose. A dieta dos animais também pode ser manipulada para causar variações nos componentes do leite, e o teor de gordura pode variar em até 3\%. Nos animais criados em sistema orgânico, a alimentação é predominantemente em pastos, enquanto que no sistema convencional a alimentação baseia-se também em grãos e bolo de soja. Verificou-se que, no inverno, o leite orgânico apresentou 2,67\% de gordura, sendo o valor mais baixo encontrado neste estudo. Esse valor foi significativamente diferente $(\mathrm{p} \leq 0,05)$ daqueles encontrados durante a primavera e o outono, isto é, 3,30 e 3,32\%, respectivamente. Os leites orgânicos avaliados neste estudo foram provenientes de pequenos laticínios, característica de leite orgânico no Brasil, em que dificilmente há processo de homogeneização, devido ao custo do equipamento. No leite não homogeneizado resfriado, a gordura, que possui densidade mais baixa, se separa da fase aquosa, sendo difícil formar uma emulsão estável. Ainda, vale salientar que algumas amostras foram congeladas para posterior análise. A junção desses fatores poderia explicar o valor abaixo do normal observado nas amostras do período de inverno. Essas diferenças não foram observadas em leite convencional, em que os valores variaram entre 3,39 e 3,49\% de gordura, sem diferença significativa. Nota-se que o leite convencional apresentou teor mais alto de gordura que o leite orgânico, independentemente da estação do ano. Por vezes, tratamentos dietéticos que elevam o teor de CLA simultaneamente reduzem o teor de gordura do leite (EIFERT et al., 2006).

Tendência oposta foi observada em relação ao teor de proteína nos leites estudados (Tabela 1). Observa-se que em leite proveniente de manejo orgânico a fração protéica variou significativamente de $3,22 \%$ (verão de 2007) a 3,42\% (outono de 2007) ( $\mathrm{p} \leq 0,05)$. Esses valores foram maiores que aqueles encontrados em leite convencional. Os teores de proteína e gordura são mais baixos durante o verão devido às mudanças na qualidade e disponibilidade da forragem. No rúmen, a produção de propionato promove a síntese de proteína, sendo que o aumento de propionato produzido é acrescido pela alimentação de forragem picada (HARDING, 1995). Não foram observados efeitos do manejo e da sazonalidade no teor de lactose dos leites orgânicos e convencionais amostrados ( $\mathrm{p} \geq 0,05)$. A lactose é o componente que menos varia no leite bovino
(GONZÁLEZ, 2001), como observado no presente trabalho (Tabela 1). Segundo Harding (1995), o conteúdo de lactose não é influenciado pela dieta, exceto através de excesso ou falta de alimentação, porém as porcentagens de lactose podem ser mais baixas durante as estações mornas, fator que acarreta menor consumo de fibras pelo animal.

A sazonalidade apresentou pouco efeito no teor de cinzas. Leites orgânicos apresentaram, em todas as estações do ano, teores de cinzas ligeiramente superiores àqueles de leite convencional, no entanto, somente no inverno houve diferença significativa. Jenness (1980) também encontrou valores superiores para cinzas no inverno.

Pode-se observar pela ANOVA apresentada na Tabela 2 que a proveniência do leite ou a estação do ano tem efeito significativo na acidez, na gordura, na proteína e nas cinzas dos leites, enquanto estes fatores simultaneamente afetam significativamente apenas acidez, gordura e cinzas ( $p \leq 0,05)$. Não foi observado, porém, efeito da origem do leite e da sazonalidade na densidade, no extrato seco, na proteína e no teor de lactose $(\mathrm{p} \geq 0,05)$. O teor do extrato seco dos leites foi influenciado pela sazonalidade $(\mathrm{p} \leq 0,05)$.

\subsection{Variação sazonal do perfil de ácidos graxos e teor de ácido linoléico conjugado}

Na Tabela 3, está apresentado o perfil de ácidos graxos existentes no leite, sendo principais os ácidos 14:0, 16:0, 18:0 e 18:1. Os resultados foram expressos em porcentagem de ácidos graxos. A variação encontrada entre as amostras dentro de uma mesma estação do ano se deve às diferentes marcas dos leites analisados, explicando os altos valores do desvio padrão.

As estações do ano e o manejo dos animais não apresentaram diferenças significativas no perfil de ácidos graxos do leite. Observa-se que o teor de ácidos graxos saturados dos leites convencionais sempre foi superior ao teor dos leites orgânicos. Dentre os ácidos graxos saturados, foram encontradas diferenças significativas quanto às estações do ano nos teores do ácido cáprico (10:0) (Tabela 3). O perfil de ácidos graxos dos leites, apresentado neste trabalho, é próximo daquele descrito por Eifert et al. (2006) para gordura de leite de vaca.

A Figura 1 apresenta a análise cromatográfica dos ácidos graxos no leite orgânico, no qual se observa a presença de CLA. Os valores de CLA obtidos para leite orgânico variaram de $0,69 \%$ (inverno de 2006) a 1,68\% (outono de 2007). A diferença entre os manejos estudados tornou-se evidente nos resultados de CLA, nos quais o leite orgânico apresentou até 2,8 vezes mais CLA que o leite convencional (outono de 2007) (Figura 2). Estes resultados correspondem aos encontrados na literatura (BERGAMO et al., 2003; EIFERT et al., 2006; JAHREIS et al., 2007).

\section{Conclusões}

Os resultados do estudo da variação sazonal da composição físico-química, perfil de ácidos graxos e teor de ácido linoléico conjugado em leites orgânicos e convencionais 
Tabela 2. Resultados da ANOVA para composição físico-química de leites integrais orgânicos e convencionais comercializados na cidade de São Paulo.

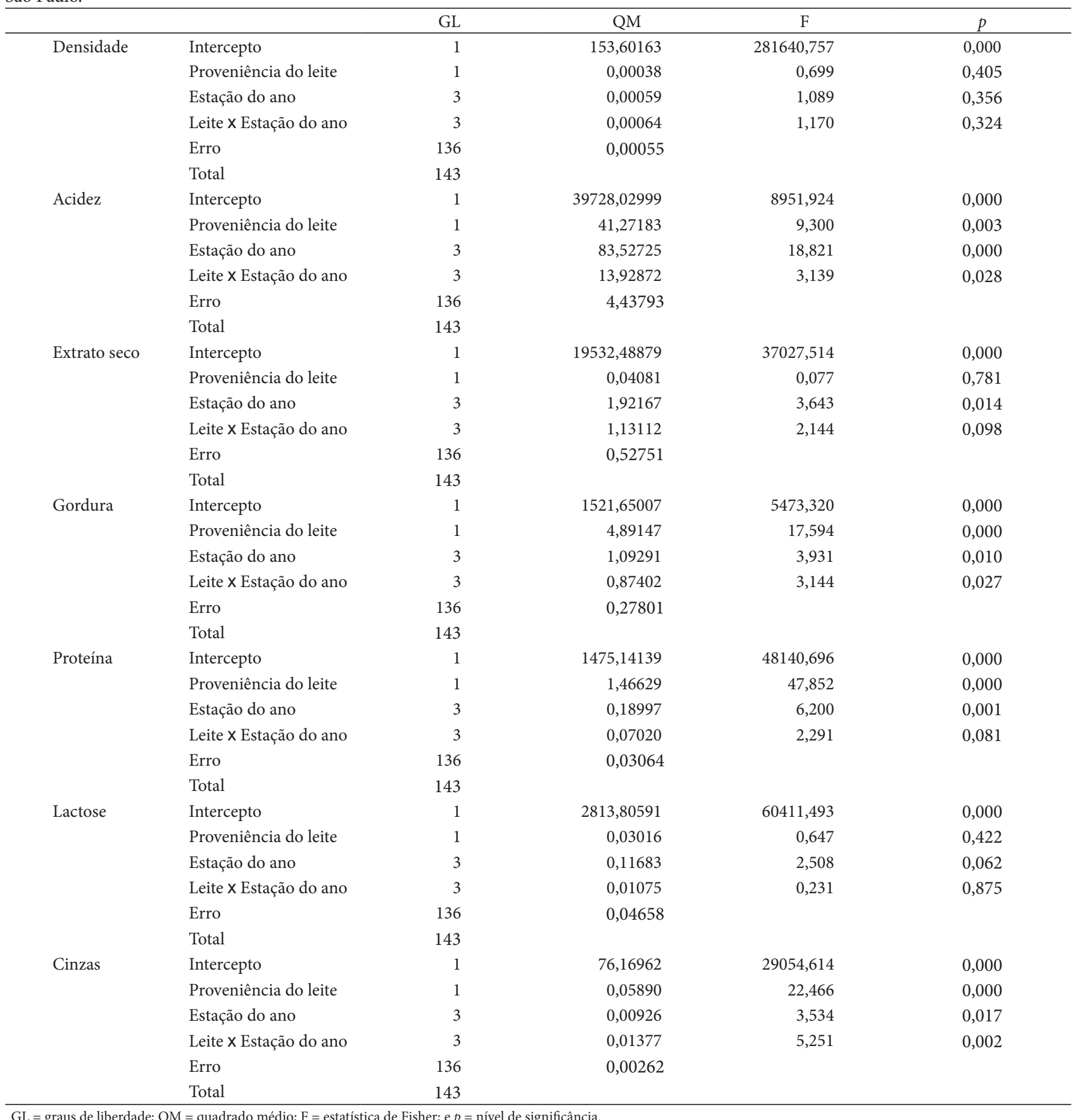

$\mathrm{GL}=$ graus de liberdade; $\mathrm{QM}=$ quadrado médio; $\mathrm{F}=$ estatística de Fisher; $\mathrm{e} p=$ nível de significância.

comercializados na cidade de São Paulo permitiram concluir que:

- A escolha do sistema de manejo (orgânico e convencional) e a sazonalidade afetam a composição química dos leites;

- O teor de proteínas em leite orgânico sempre apresentou maiores teores que em convencional;
- O teor de gordura do leite foi influenciado pelo sistema de manejo, sendo que os leites do sistema orgânico apresentaram menores teores de gordura e menores percentuais de gordura saturada que o sistema convencional; e

- O teor de ácido linoléico conjugado (CLA) dos leites provenientes do sistema orgânico foi superior ao dos leites provenientes do sistema convencional. 
Tabela 3. Composição (\%) de ácidos graxos ${ }^{1}$ de leite integral pasteurizado orgânico e convencional comercializados na cidade de São Paulo.

\begin{tabular}{|c|c|c|c|c|c|c|c|c|}
\hline \multirow[b]{2}{*}{ Ácidos graxos } & \multicolumn{2}{|c|}{ Inverno/06 } & \multicolumn{2}{|c|}{ Primavera/06 } & \multicolumn{2}{|c|}{ Verão/07 } & \multicolumn{2}{|c|}{ Outono/07 } \\
\hline & Orgânico & Convencional & Orgânico & Convencional & Orgânico & Convencional & Orgânico & Convenciona \\
\hline $\mathrm{C} 4: 0$ & $4,42^{\mathrm{d}} \pm 0,61$ & $1,59^{\mathrm{abc}} \pm 0,24$ & $2,69^{\mathrm{bcd}} \pm 0,29$ & $4,12^{\mathrm{d}} \pm 0,67$ & $2,85^{\mathrm{cd}} \pm 0,40$ & $3,48^{\mathrm{cd}} \pm 0,45$ & $0,34^{a} \pm 0,00$ & $0,79^{a} \pm 0,36$ \\
\hline C6:0 & $3,06^{c} \pm 0,83$ & $2,78^{\mathrm{bc}} \pm 0,46$ & $2,05^{\mathrm{abc}} \pm 0,56$ & $3,29^{c} \pm 0,38$ & $1,97^{\mathrm{abc}} \pm 0,35$ & $2,77^{\mathrm{bc}} \pm 0,43$ & $0,79^{\mathrm{a}} \pm 0,33$ & $1,36^{\mathrm{ab}} \pm 0,48$ \\
\hline C10:0 & $2,81^{\mathrm{ab}} \pm 0,97$ & $3,81^{\mathrm{ab}} \pm 0,85$ & $2,92^{\mathrm{ab}} \pm 1,17$ & $4,35^{\mathrm{b}} \pm 0,26$ & $2,61^{\mathrm{ab}} \pm 0,48$ & $3,57^{\mathrm{ab}} \pm 0,40$ & $2,07^{\mathrm{a}} \pm 0,59$ & $2,50^{\mathrm{ab}} \pm 0,26$ \\
\hline C10:1 & - & $0,43^{a} \pm 0,15$ & $0,19^{\mathrm{a}} \pm 0,08$ & $0,35^{\mathrm{a}} \pm 0,09$ & - & $0,46^{\mathrm{a}} \pm 0,05$ & - & $0,01^{a} \pm 0,00$ \\
\hline C14:1 & $0,87^{\mathrm{a}} \pm 0,05$ & $1,45^{a} \pm 0,05$ & $1,34^{\mathrm{a}} \pm 0,16$ & $1,45^{\mathrm{a}} \pm 0,21$ & $1,57^{a} \pm 0,12$ & $1,32^{\mathrm{a}} \pm 0,19$ & $1,41^{a} \pm 0,26$ & $1,30^{\mathrm{a}} \pm 0,31$ \\
\hline C15:0 & $1,66^{\mathrm{b}} \pm 0,25$ & $1,33^{\mathrm{ab}} \pm 0,18$ & $1,44^{\mathrm{ab}} \pm 0,11$ & $1,21^{a} \pm 0,13$ & $1,52^{\mathrm{ab}} \pm 0,13$ & $1,34^{\mathrm{ab}} \pm 0,04$ & $1,54^{\mathrm{ab}} \pm 0,06$ & $1,25^{a} \pm 0,15$ \\
\hline C16:0 & $28,20^{\mathrm{ab}} \pm 2,10$ & $31,21^{\mathrm{b}} \pm 1,13$ & $29,04^{\mathrm{ab}} \pm 1,46$ & $28,97^{\mathrm{ab}} \pm 0,94$ & $27,10^{a} \pm 0,53$ & $29,83^{\mathrm{ab}} \pm 0,78$ & $28,67^{\mathrm{ab}} \pm 0,55$ & $30,78^{b} \pm 1,51$ \\
\hline C16:1 & $1,70^{\mathrm{a}} \pm 0,23$ & $1,74^{\mathrm{a}} \pm 0,06$ & $1,81^{\mathrm{a}} \pm 0,28$ & $1,47^{\mathrm{a}} \pm 0,06$ & $1,70^{\mathrm{a}} \pm 0,14$ & $1,49^{\mathrm{a}} \pm 0,17$ & $1,76^{\mathrm{a}} \pm 0,05$ & $1,80^{\mathrm{a}} \pm 0,17$ \\
\hline C17:0 & $0,77^{a} \pm 0,20$ & $0,54^{\mathrm{a}} \pm 0,06$ & $0,80^{\mathrm{a}} \pm 0,09$ & $0,21^{a} \pm 0,00$ & $1,06^{\mathrm{a}} \pm 0,07$ & $0,67^{\mathrm{a}} \pm 0,06$ & $0,69^{\mathrm{a}} \pm 0,34$ & $0,72^{a} \pm 0,03$ \\
\hline C18:2 & $1,88^{\mathrm{ab}} \pm 0,71$ & $3,02^{\mathrm{abc}} \pm 0,97$ & $2,09^{\mathrm{ab}} \pm 0,68$ & $3,96^{\mathrm{bc}} \pm 0,56$ & $1,30^{\mathrm{a}} \pm 0,32$ & $3,58^{\mathrm{bc}} \pm 0,61$ & $1,77^{\mathrm{ab}} \pm 0,51$ & $4,49^{c} \pm 1,39$ \\
\hline C18:3 & $0,64^{\mathrm{a}} \pm 0,05$ & $0,71^{\mathrm{a}} \pm 0,03$ & $0,19^{\mathrm{a}} \pm 0,00$ & $0,55^{\mathrm{a}} \pm 0,25$ & $0,22^{\mathrm{a}} \pm 0,00$ & $0,67^{\mathrm{a}} \pm 0,03$ & $0,49^{\mathrm{a}} \pm 0,16$ & $0,60^{\mathrm{a}} \pm 0,45$ \\
\hline CLA & $0,69^{a} \pm 0,12$ & $0,71^{a} \pm 0,15$ & $0,93^{\mathrm{ab}} \pm 0,29$ & $0,55^{\mathrm{a}} \pm 0,08$ & $1,61^{\mathrm{b}} \pm 0,48$ & $0,67^{a} \pm 0,14$ & $1,68^{b} \pm 0,53$ & $0,60^{a} \pm 0,09$ \\
\hline Saturados & $68,55^{a} \pm 6,53$ & $70,39^{a} \pm 2,48$ & $65,41^{\mathrm{a}} \pm 6,95$ & $70,93^{a} \pm 1,29$ & $64,67^{\mathrm{a}} \pm 1,42$ & $69,19^{\mathrm{a}} \pm 1,35$ & $61,76^{a} \pm 3,45$ & $66,76^{a} \pm 0,38$ \\
\hline Monoinsaturados & $28,25^{\mathrm{ab}} \pm 5,97$ & $25,44^{\mathrm{ab}} \pm 1,30$ & $31,38^{\mathrm{ab}} \pm 6,39$ & $24,15^{\mathrm{a}} \pm 0,31$ & $32,19^{\mathrm{ab}} \pm 0,44$ & $25,93^{\mathrm{ab}} \pm 0,49$ & $34,32^{\mathrm{b}} \pm 2,02$ & $27,64^{\mathrm{ab}} \pm 1,42$ \\
\hline Poliinsaturados & $3,20^{\mathrm{a}} \pm 0,58$ & $4,17^{\mathrm{a}} \pm 1,20$ & $3,21^{\mathrm{a}} \pm 1,09$ & $4,92^{\mathrm{a}} \pm 0,98$ & $3,13^{\mathrm{a}} \pm 0,44$ & $4,87^{\mathrm{a}} \pm 0,49$ & $3,93^{\mathrm{a}} \pm 1,41$ & $5,60^{\mathrm{a}} \pm 1,70$ \\
\hline
\end{tabular}

${ }^{1}$ Valores dados como médias com desvio padrão; ${ }^{\text {ab, }, c}$ médias $(\mathrm{n}=18)$ na mesma linha com letras diferentes são significativamente diferentes $p \leq 0,05$; e -- = traços.

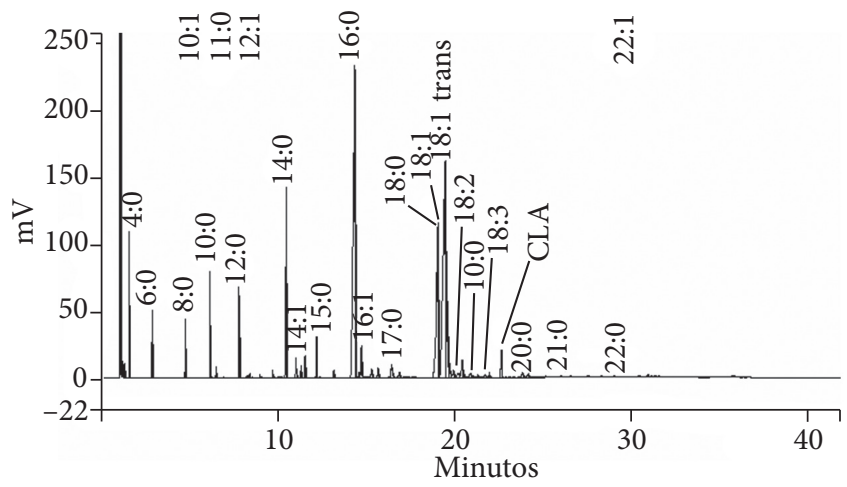

Figura 1. Perfil de ácidos graxos e pico do ácido linoléico conjugado obtido por cromatografia gasosa de ésteres metílicos de leite orgânico.

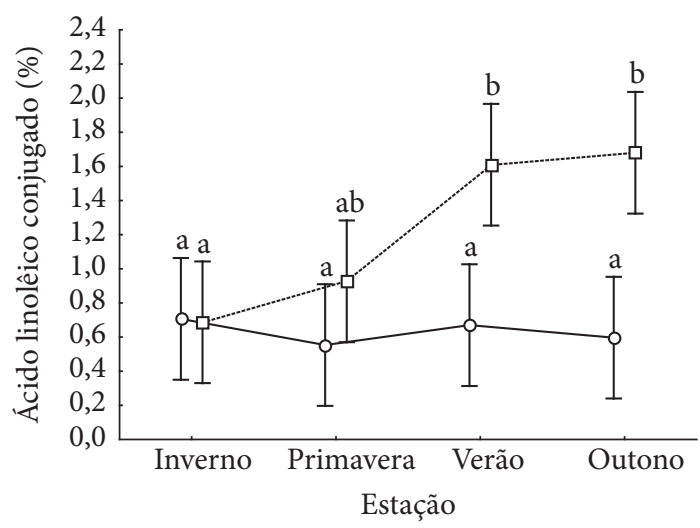

Figura 2. Comportamento do teor de ácido linoléico conjugado em leite convencional ( $($ ) e orgânico $(\square)$. Médias $(n=3)$ com letras diferentes são significativamente diferentes; $\mathrm{p} \leq 0.05$.

\section{Agradecimentos}

Agradecemos ao CNPq pela Bolsa de Iniciação Científica de Michele G. N. Fanti e à FAPESP pelo apoio financeiro para o desenvolvimento deste trabalho (Processos 05/57599-5 e 07/51904-6).

\section{Referências bibliográficas}

ASSOCIATION OF OFFICIAL ANALYTICAL CHEMISTS. Official methods of analysis of Association of Official Analytical Chemists. 16 ed. Arlington: AOAC, 1995. cap. 4.2, p. 5.

AMERICAN OIL CHEMISTS' SOCIETY. Official methods and recommended practices of the American Oil Chemistis' Society. 4 ed. Champaign: AOCS, 1997.

AMIOT, J. Ciencia y tecnologia de la leche: principios y aplicaciones. Zaragoza: Acribia, 1991.p. 1-30.

BERGAMO, P.; FEDELE, E.; IANNIBELLI, L.; MARZILLO, G. Fat soluble vitamin contents and fatty acid composition in organic and conventional Italian dairy products. Food Chemistry, v. 82, n. 4 , p. 625-631, 2003.

BRASIL. Lei n.10.831, de 23 de dezembro de 2003a. Dispõe sobre a agricultura orgânica. In: IBD CERTIFICAÇÕES. Diretrizes e Legislação. Decreto da Lei 10.831 de Produtos Orgânicos. Disponível em: http://www.ibd.com.br/Downloads/DirLeg/ Legislacao/05-Lei_10831_2003.pdf. Acesso em: 4 Jun. 2007.

BRASIL. Ministério da Agricultura, Pecuária e Abastecimento. Legislação. SISLEGIS - Sistema de Consulta à Legislação. Instrução Normativa n.68, de 12 de dezembro de 2006. Oficializa os métodos analíticos oficiais físico-químicos, para controle de leite e produtos lácteos, em conformidade com o anexo desta Instrução Normativa, determinando que sejam utilizados nos Laboratórios Nacionais Agropecuários. Disponível em: http://extranet.agricultura.gov.br/ 
sislegis-consulta/consultarLegislacao.do?operacao=visualizar\&id= 17472. Acesso em: 14 Fev. 2008.

BLOCK, E. Nutrição de vacas leiteiras e composição do leite. In: ENCONTRO ANUAL DO CONSELHO BRASILEIRO DE QUALIDADE DO LEITE, 1, 2000, Curitiba. Anais... Curitiba: Centro Integrado dos empresários e trabalhadores das Indústrias do Paraná, FIEP, 2000. p. 85-88.

CAMPBELL, W.; DRAKE, M. A.; LARICK, D. K. The impact of fortification with conjugated linoleic acids (CLA) on the quality of fluid milk. Journal of Dairy Science, v. 86, n. 1, p. 43-51, 2003.

CARIS-VEYRAT, C. et al. Influence of organic versus conventional agricultural practice on the antioxidant microconstituent content of tomatoes and derived purees; consequences on antioxidant plasma status in humans. Journal of Agricultural and Food Chemistry, v. 52, n. 21, p. 6503-6509, 2004.

DAROLT, M. R. Pecuária orgânica: procedimentos básicos para um bom manejo da criação. In: Planeta Orgânico. Trabalhos Publicados. Disponível em: http://www.planetaorganico.com.br/ daroltpec1.htm. Acesso em: 14 Ago. 2007.

DÍAZ GAMBOA, O. W.; GIOIELLI, L. A. Lípidos estructurados obtenidos por interesterificación química y enzimática a partir de aceite de pescado y grasa de semilla de palma. Grasas y Aceites, v. 54, n. 2, p. 161-168, 2003.

EIFERT, E. C. et al. Perfil de ácidos graxos e conteúdo de ácido linoléico conjugado no do leite de vacas alimentadas com a combinação de óleo de soja e fonte de carboidratos na dieta. Revista Brasileira de Zootecnia, v. 35, n. 4, p. 1829-1937, 2006.

FELSOT, A. S.; ROSEN, J. D. Comment on comparison of the total phenolic and ascorbic acid content of freeze-dried and air-dried marionberry, strawberry, and corn grown using conventional, organic, and sustainable agricultural practices. Journal of Agricultural and Food Chemistry, v. 52, n. 1, p. 146-149, 2004.

GONZÁLEZ, F. H. D. Composição bioquímica do leite e hormônios da lactação. In: GONZÁLEZ, F. H. D.; DÜRR, J. N.; FONTANELI, R. S. Uso do leite para monitorar a nutrição e o metabolismo de vacas leiteiras. Porto Alegre: UFRGS, 2001. p. 5-21. Disponível em: http://www6.ufrgs.br/bioquimica/extensao/anais_2001.pdf. Acesso em: 11 Jun. 2007.

HARDING, F. Compositional quality: milk quality. Glasgow: Blackie Academic Professional, 1995. 165 p.

HEATON, S. Organic farming: food quality and human health. Bristol: Soil Association, 2001. p. 11-17.

HOLMES, C. W.; WILSON, G. F. Produção de leite a pasto. Campinas: Instituto Campineiro de Ensino Agrícola, 1989. 708p.
INSTITUTO ADOLFO LUTZ. Normas analíticas do Instituto Adolfo Lutz: métodos químicos e físicos para análise de alimentos. 3 ed. São Paulo: Imprensa Oficial do Estado de São Paulo, 1985. v. $1,533 \mathrm{p}$.

INTERNATIONAL ORGANIZATION FOR STANDARDIZATION. ISO/DIS 15884: Milk fat: preparation of fatty acid methyl esters. Geneve: ISO, 2000.

INTERNATIONAL ORGANIZATION FOR STANDARDIZATION. ISO/DIS 14156: Milk and milk products: extraction methods for lipids and liposoluble compounds. Geneve: ISO, 2000.

JAHREIS, G.; FRITSCHE, J.; STEINHART, H. Conjugated linoleic acid in milk fat: high variation depending on production system. Nutrition Research, v. 17, n. 9, p. 1479-1484, 1997.

JENNESS, R. Compositions and characteristics of goat's milk: review 1968-1979. Journal of Dairy Science, v. 63, n. 10, p. 1605-1630, 1980.

KÜHLSEN, N. et al. Trans fatty acids: scientific progress and labeling. Bulletin of the International Dairy Federation, n. 393, p. 1-25, 2005.

LUUKKONEN, J. et al. The effect of a protective culture and exclusion of nitrate on the survival of enterohemorrhagic E.coli and Listeria in Edam cheese made from Finnish organic milk. International Dairy Journal, v. 15, n. 15, p. 449-457, 2005.

MATTILA SANDHOLM, T.; SAARELA, M. Functional dairy products. Boca Raton: CRC Press; Cambridge: Woodhead Publisinhg, 2003. 395 p. (Woodhead Publisinhg in Food Science and technology).

PRANDINI, A. et al. Different level of conjugated linoleic acid (CLA) in dairy products from Italy. Journal of Food Composition and Analysis, v. 20, n. 6, p. 472-479, 2007.

ROSATI, A.; AUMAITRE, A. Organic dairy farming in Europe. Livestock Production Science, v. 90, n. 1, p. 41-51, 2004.

SEBEDIO, J. L.; GNÄDIG, S.; CHARDIGNY, J. M. Recent advances in conjugated linoleic acid research. Current Opinion in Clinical Nutrition and Metabolic Care, v. 2, n. 6, p. 499-506, 1999.

TOLEDO, P.; ANDRÉN, A.; BJÖRCK, L. Composition of raw milk from sustainable production systems. International Dairy Journal, v. 12, n. 1, p. 75-80, 2002.

VON BORELL, E.; SORENSEN, J. T. Organic livestock production in Europe: aims, rules and trends with special emphasis on animal health and welfare. Livestock Production Science, v. 90, n. 1, p. 3-9, 2004. 